\title{
Exploiting Interchangeabilities for Case Adaptation
}

\author{
Nicoleta Neagu and Boi Faltings \\ Artificial Intelligence Laboratory (LIA), \\ Computer Science Department, Swiss Federal Institute of Technology (EPFL) \\ CH-1015 Ecublens, Switzerland \\ \{neagu, faltings\}@lia.di.epfl.ch http://liawww.epfl.ch/
}

\begin{abstract}
While there are many general methods for case retrieval, case adaptation usually requires problem-specific knowledge and it is still an open problem. In this paper we propose a general method for solving case adaptation problems for the large class of problems which can be formulated as Constraint Satisfaction Problems. This method is based on the concept of interchangeability between values in problem solutions. The method is able to determine how change propagates in a solution set and generate a minimal set of choices which need to be changed to adapt an existing solution to a new problem.

The paper presents the proposed method, algorithms and test results for a resource allocation domain.
\end{abstract}

Keywords: case-based reasoning, case adaptation, constraint satisfaction problems, interchangeability.

\section{Introduction}

Case-based reasoning (CBR) for solving problems can be broken down into two important steps: case retrieval and case adaptation [1]. While there are many general methods for case retrieval, solutions to case adaptation remain highly domain specific, requiring detailed problem-specific knowledge.

Although it may be unrealistic to hope for generic methods to solve all adaptation problems, one class of problems which may be addressed is problems which can be formulated as Constraint Satisfaction Problems (CSPs) [2]. CSPs consist of a number of choices that need to be made (variables), each of which has an associated number of options (the variable domain) and a set of relationships between choices (constraints). A valid solution to a CSP is an assignment of a value to each variable from its domain with the total set of assignments respecting all the problem constraints. The CSP model can therefore be applied to a very wide range of problems [3], well known examples are: diagnosis [4], planning [5], scheduling [6], robot control [7] and configuration [8].

This flexibility makes CSP problems a valuable class of problems for CBR adaptation methods. Solutions which can be shown to apply to CSP type problems can be applied to a wide range of problems.

This paper presents a method based on the concepts of interchangeability [9] and neighbourhood partial interchangeability (NPI) [10]. These methods can be used to capture equivalence among values of a variable in a discrete CSP (original interchangeabil- 
ity) and localise the effect of modifications to some variables and determine relations between close solutions.

These techniques are applied and extended to case adaptation to enable a CBR system to determine a minimal change in the solution of the retrieved case. The main contributions of this paper are:

- A model for the application of Interchangeability techniques to a large class of CBR adaptation problems (Section 3).

- An algorithm for computing minimal neighbourhood partial interchangeability; it determines how change propagates in a solution set and generates a minimal set of choices which need to be changed in order to adapt an existing solution to a new problem. (Section 3.4).

- Test results and initial analysis of the effectiveness of the adaptation technique (Section 4).

Additionally, in Section 2 we recall the main definitions of interchangeability that are of interest to us while conclusions and further work can be found in Section 6.

\section{Definitions}

Definition 1 (CSP). A CSP is defined by $P=(V, D, C)$, where $V=\left\{V_{1}, V_{2}, \ldots, V_{n}\right\}$ is the set of variables, $D=\left\{D_{V 1}, D_{V 2}, \ldots, D_{V n}\right\}$ the set of domains (i.e., sets of values) associated with the variables, and $C$ is the set of constraints that apply to the variables.

In other words, Constraint Satisfaction Problems (CSPs) involve finding values for variables subject to constraints on which combination of values are permitted. The work presented here is currently restricted to problems which can be modelled as discrete binary CSPs. These are problems where each domain contains a finite set of discrete values and constraints are never between more than two variables. Although this is a restriction, this is the most common class of CSPs and covers a large range of problems. In these work it was considered that the constraints are hard, where for specific values assigned to the variables involved in the constraint, the constraint can be satisfied or not. In further work, we intend to investigate how our algorithms for detecting minimal changes apply CSP with fuzzy constraints [11], where different tuples satisfy the given constraint to a different degree.

The concept of Interchangeability formalises equivalence relations among objects, respectively the values of the variables in a CSP. The concept of interchangeability was first introduced by Freuder in [9]. Among others, Freuder defines the following three kinds of interchangeability:

Definition 2 (Full Interchangeability - FI). Values $V_{i}=a$ and $V_{i}=b$ are fully interchangeable if for any solution where $V_{i}=a$, there is an otherwise identical solution where $V_{i}=b$, and vice versa.

This means that by exchanging values a and $\mathrm{b}$ for variable $V_{i}$ in a given solution, the solution will remain a valid solution of the CSP (without requiring changes to the other variable assignments). There is no efficient general algorithm for computing FI values in a CSP; this might require computing all solutions [9]. 
Definition 3 (Neighbourhood Interchangeability - NI). Values $V_{i}=a$ and $V_{i}=b$ are neighbourhood interchangeable if for every constraint involving $V_{i}$, for every tuple that admits $V_{i}=a$ there is otherwise an identical tuple that admits $V_{i}=b$, and viceversa.

Neighbourhood Interchangeability considers only local interactions and thus can be efficiently computed. Freuder proposes a polynomial-time algorithm for computing NI values [9].

Definition 4 (Partial Interchangeability - PI). Values $V_{i}=a$ and $V_{i}=b$ are partially interchangeable with respect to a set of variables $S$ if for any solution where $V_{i}=a$, there is another solution where $V_{i}=b$ which otherwise differs only in values assigned to variables in $S$, and vice versa.

Partial Interchangeability exploits the idea that values for a subset of variables from the CSP may differ among themselves, but stay fully interchangeable with the rest of the CSP. We can therefore identify groups of partial solutions. Equivalent solutions can be generated by modifying the values of the indicated variables only.

Again, there is no efficient algorithm for computing PI set. A localised algorithm has however been proposed for computing Neighbourhood Partial Interchangeability (NPI) sets of variables with the corresponding NPI values [10].

Definition 5 (Neighbourhood Partial Interchangeability - NPI). Values $V_{i}=a$ and $V_{i}=b$ are neighbourhood partial interchangeable (NPI) with respect to a set of variables $S$ if for every constraint between $V_{i}$ and the neighbourhood of set $S$, for every tuple that admits $V_{i}=a$ there is otherwise an identical tuple that admits $V_{i}=b$, and vice-versa, (where this change can affect the variables from set $S$ ), while the same condition applies also for all the other variables from $S$.

NPI is a localised form of PI where the propagation of the change is computed by looking 'through' the neighbourhood of the set $\mathrm{S}$.

NPI interchangeability classes are computed by the use of the Joint Discrimination Tree (JDT) algorithm, as described in [10], where the annotations of the JDT of a set S $=\left\{V_{1}, V_{2}, \ldots, V_{k}\right\}$ contains NPI values as follows:

$$
\begin{aligned}
\mathrm{NPI}(\mathrm{S})=\{ & \left\{\left(V_{1}, d_{m 1}\right),\left(V_{2}, d_{m 2}\right), \ldots,\left(V_{k}, d_{m k}\right)\right\} \\
& \text { such that } \left.\left(\forall 1 \leq \mathrm{i} \leq \mathrm{k}, d_{m i} \neq \Phi\right) \bigwedge\left(\exists 1 \leq \mathrm{i} \leq \mathrm{k},\left|d_{m i}\right|>1\right)\right\}
\end{aligned}
$$

Definition 6 (Minimal Neighbourhood Partial Interchangeability Set - MNPIS). For $\forall V_{i}$ belonging to a NPI set $S, \forall v_{i j k}, v_{i j l} \in d_{i j}$ in a NPI set exchanging $v_{i j k}$ with $v_{i j l}$ implies $\exists V_{n} \in S$ which has to change its value (only in the domain $d_{n j}$ of the interchangeability class of the current NPI set) such that the constraint in the set $S$ are satisfied and the solution maintained.

In other words, exchanging the value of one variable $V_{i}$ contained in a NPI set $\mathrm{S}$, has to affect at least one of the other variables of the set $S$.

Definition 7 (Minimum Neighbourhood Partial Interchangeability Set - mNPIS). A minimum NPI set for a variable $V_{i}$ is the minimal NPI set which contains the minimum number of variables, including the starting variable $V_{i}$. (take here into consideration that the sets are constructed starting from a variable and enlarging its environment by following its constraints and including the neighbors in the constructed set) 


\section{Adaptation Model based on Interchangeability}

In previous work [12], the authors applied a restricted interchangeability framework to case adaptation problems in car configuration. This previous approach was able to identify NI values for CSP values and therefore determine individal choices in a solution which could be adapted. The work here extends this idea to include adaptation over complete NPI sets to find minimal sets of variables which might be changed to generate adapted solutions. The framework is illustrated through an example application to a generic resource allocation problem.

The resource allocation problem used is defined as a CSP in the following way:

- tasks $\left(T_{1}, T_{2}, \ldots, T_{n}\right)$ are considered as the variables of the CSP where their values are resources.

- domains of variables are sets of resouces by which the tasks can be executed.

- constraints among variables denote mutual exclusion with respect to the values. That means that two tasks overlapping in time cannot be carried out by the same resource.

Since the main contribution of this work relates to the adaptation step of the CBR process, retrieval is done use a simple metric which picks out the closest previous case.

\subsection{CBR Model}

The framework presented in the figure 1 solves new resource allocation problems by retrieving and adapting previous solutions. The problem solving process proceeds as follows:

1. A new problem (defined in terms of tasks and resources to allocate as above) arrives in the system.

2. The problem is matched to a single previous case.

3. The adaptation phase of the process therefore receives the following inputs:

- The solution of the retrieved case.

- The corresponding CSP retrieved from the CSP base according to the tasks contained in the new problem.

- The differences between the problem at hand and the one expressed in the indexing parameters of the retrieved case ${ }^{1}$ (see Figure 1).

4. The adaptation module applies the minimal NPI algorithm (Section 3.4) to this input to generate the closest solution to the new problem.

The adaptation phase of the process therefore has an extra form of information available to it which is the CSP model corresponding to the new problem. The domain knowledge is represented as a CSP problem. It is this that allows us to apply the interchangeability algorithms. The most common algorithm for performing systematic

\footnotetext{
${ }^{1}$ The requirements can also be imposed by the user.
} 


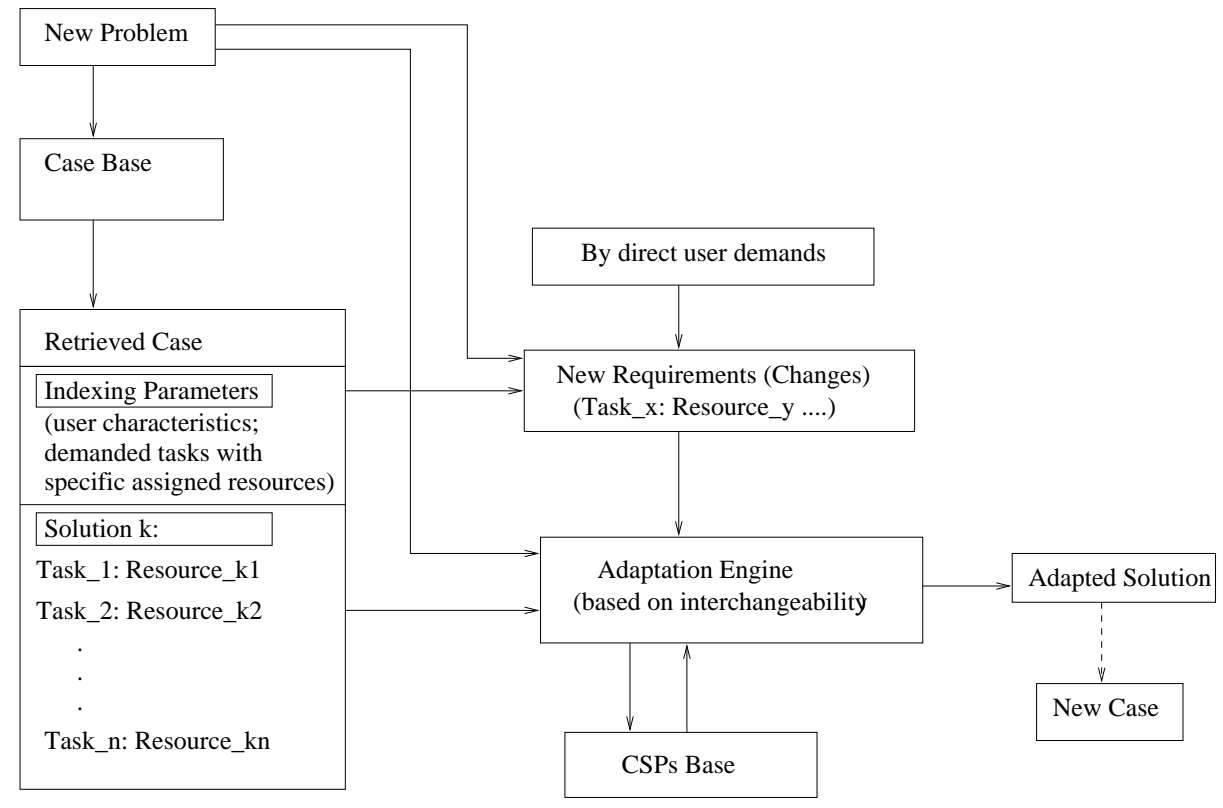

Fig. 1. Adaptation Model

search in a CSP is backtracking but in large CSPs it might be expensive. Thus, inffering a new solution from an already known one by studying local changes based on interchangeability, have a linear cost and can be used as a reliable adaptation method.

For the tasks with their corresponding exchanging values, provided by the new requirements module, the adaptation engine applies the minimal NPI algorithm (Section 3.4) in order to find the NI or NPI values for adapting solutions. For an input as single task, it might find that the values proposed to be exchanged are NI, and thus the new solution keeps all the same values for all the other tasks and exchanges only the NI values of the task asked to be changed. In other cases the algorithm might find an NPI set of variables which have to be changed in order to get a solution for the new requirements. Thus the constraints between the variables of the NPI set have to be solved, while all the others variables of the solution stay unchanged. We notice that the computational effort is here restricted to the NPI set and one does not have to solve the whole problem from scratch. If the minimal NPI set finding algorithm does not find any NPI set, it means that there are no solutions for the new requirements in the limited threshold of number of variables which might be changed imposed in the minimal NPI algorithm. In this situation, it might be necessary to solve the problem from scratch.

In the CSP base we store the knowledge about the domain in the form of CSPs. In our previous work, the car configuration system [12] there we had only one CSP which modeled all possible configurations. For increasing the generality, we propose now to represent the knowledge by several CSP, which modell different resource allocation problems. 


\subsection{Example of Applying Neighborhood Interchangeability (NI) to Case Adaptation.}

As presented in previous work, the simplest way to apply interchangability to case adaption is to find NI sets of values for a variables of the CSP. Figure 2 shows an example of this for a resource allocation problem. The resource allocation problem is modelled as a discrete CSP in which the constraints are binary and denotes mutual exclusion with respect to the values. The nodes of the CSP represents the tasks to be executed, and their values are sets of resources by which these tasks can be executed. Arcs links tasks that overlap in time and have at least one resource in common.
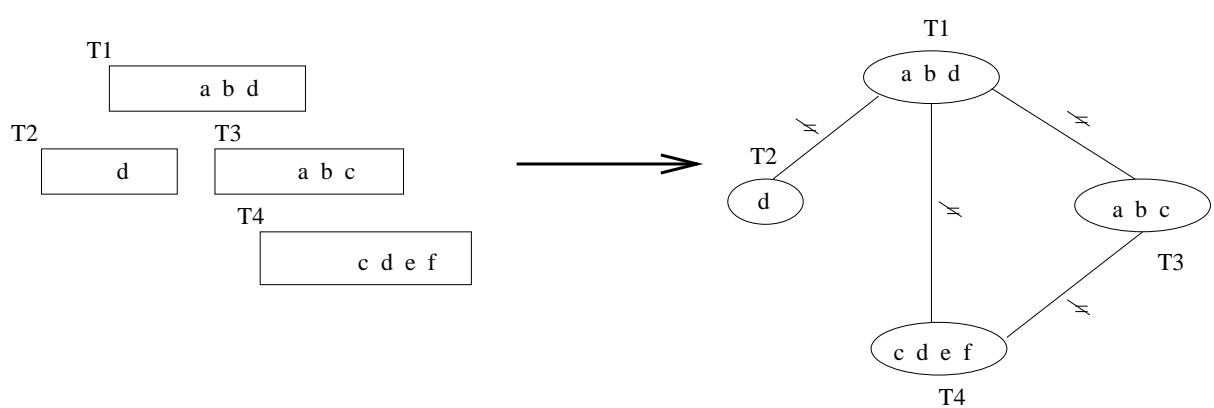

Fig. 2. Simple example of how resource allocation is modelled as CSP

Following the sequence described in the previous section a new problem might match a solution such as:

Sol1 $=\{\mathrm{T} 1=\mathrm{a}, \mathrm{T} 2=\mathrm{d}, \mathrm{T} 3=\mathrm{b}, \mathrm{T} 4=\mathrm{e}\}$

but impose the new requirements to allocate resource $\mathrm{f}$ to task T4. Normally this requirement could have a knock on effect on all or many other choices but in this case the values e and $f$ of variable T4 are NI. Thus the exchange does not affect the other variables and it stays a solution of the CSP (and hence a valid solution to the whole problem). Formulation of the problem as a CSP and applying the algorithm for detecting NI allows us to detect and prove this (which may be non-trivial in other representations).

\subsection{Example of Applying Neighborhood Partial Interchangeability (NPI) to Case Adaptation.}

Applying NI is useful but only allows single values to be exchanged in a solution. This makes a very strong requirement that zero other choices are impacted by changing an assignment. NPI is a weaker form of NI and thus more frequent in any given solution since several interdependent choices can be varied together while leaving the rest of the problem unchanged. The consequence is to isolate the effect of modification to a subproblem of the CSP. It identifies qualitatively equivalent solutions which can be generated by modifying the values of the indicated variables only. 
Since NPI is more frequent in a solution, it increases the flexibility and utility of the adaptation module. The example given in Figure 2 illustrates how the adaptation module works when using NPI interchangeability.

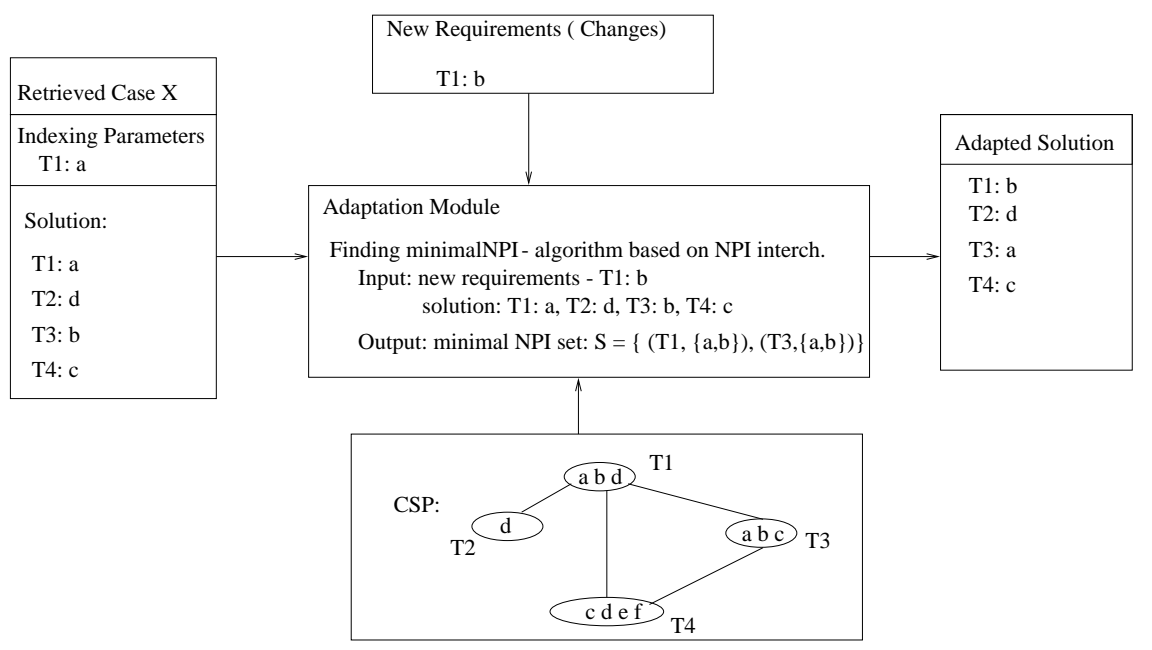

Fig. 3. Example of how the adaptation module use the NPI intercahgeability for case adaptation

The Adaptation module receives as input the solution of the retrieved case, the CSP corresponding to the current solution and of course the requirements which it has to adapt according to the requirements from the user or not matching with the indexing parameters. Indexing parametes contain previous user requirements (tasks with the corresponding resources). Similarity measure used in computing the closest case is only on simple matching between the new requirements and the previous requirements which are now the indexing parametes of the case. The differences between requirements $(\mathrm{T} 1=\mathrm{a}$ have to become $\mathrm{T} 1=\mathrm{b})$ gives the input for the minimal NPI algorithm Section 3.4.

As we can see in figure 3, by 'adapting' the task $\mathrm{T} 1$ from resource a to resource $\mathrm{b}$ we have to change also the task $\mathrm{T} 3$ from resource $\mathrm{b}$ to resource a to stay a solution, while all the other tasks remain the same. Change across two variable is therefore detected as leaving the rest of the solution valid. Although this is a simple example, the NPI algorithm can be used to detect invariance over subproblems of size $\mathrm{N}$. The system also works when several changes are required at once.

Once the NPI sets have been identified the adaptation module can check to see if it can find a valid solution, which respects the changes imposed by the new problem w.r.t. the retrieved case.

The key to being able to apply NPI set information is the algorithm for detecting these sets in the solution case to be adapted - this algorithm is presented in the next section. 


\subsection{Algorithm for finding minimal NPI sets}

The method applied for detecting NPI sets uses an algorithm which was adapted from the Joint Discrimination Tree (JDT) for computing the NPI values proposed by Choueiry and Noubir in [10]. As it is an important base to our algorithm we recall the JDT algorithm below. The complexity of this algorithm is $\mathrm{O}\left(\mathrm{s}(\mathrm{n}-\mathrm{s}) d^{2}\right)$, where $\mathrm{n}$ is the size of CSP, $s$ is the size of set S for which we compute the JDT and d the size of the largest domain.

Algorithm 1: JDT for $\mathrm{S}=\left\{V_{1}, V_{2}, \ldots, V_{k}\right\}\left(D_{V i}, \operatorname{Neigh}(\mathrm{S})\right)$ :

Create root of the Joint Discrimination tree

Repeat for each variable $V_{i} \in \mathrm{S}$

Repeat for each value $v_{i l} \in D_{V i}$

Repeat for each variable $V_{j} \in \operatorname{Neigh}(\mathrm{S})$

Repeat for each value $v_{j k} \in D_{V j}$

If there is a child node coresponding to ' $V_{j}=v_{j k}$ '

Then move to it,

Otherwise, construct such a node and move to it.

Add ' $V_{i}, v_{i l}$ ' to annotation of the node (or root)

Go back to the root of the discrimination tree.

The JDT algorithm is applied to a set $\mathrm{S}$ of variables in order to identify how these variables (when considered together and regardless of the constraints that apply among them) interact through the neighborhood with the rest of the CSP.

In the adaptation process our main interest is in seeing how much change propagates through the CSP when values are substituted for a variable. The objective is therefore to identify how many variables must change for a solution to remain valid after adaptation. The basic method is as follows:

- Start by constructing the Discrimination Tree (DT), which is the JDT applied to a set which contains a single variable, for the given values which we have to exchange.

- If the values are not in the annotation of the same branch, we reconstruct the JDT of a set formed by the starting variable and the variables found to make the difference in the branches for the considered values.

- Check if the actual JDT contains NPI values: if there exist annotations of the branches which contain at least one value for each variable from the input set and if there exists at least one variable which has at least two values (see Definition 5)

- If this is not the case, we try further to reconstruct the JDT for a new set S which includes the former $\mathrm{S}$ and the variables which make difference between the branches and separate some variables from the considered set to arrive with values in the annotation of the branch which contains the values of starting variable.

For the moment we have as a heuristic for choosing between branches: the algorithm selects the branch with the smallest differences to the refering branch and contains the starting variable with its set of pair values. So the basic principle is: whenever two values are not NPI values, we see where the branches in the tree differ. Those are variables one need to include in the set S (and thus take out of the tree) in order to create the NPI. 
The algorithm was further extended so it could be applied to a set of starting variables with their corresponding pair of values in order to identify minimal NPI set. ${ }^{2}$ In the following algorithm, the idea stays the same: we try to bring in the annotation of the same branch all the variables from the input with the corresponding values-pairs by including in the set the variables which make the difference between the branches.

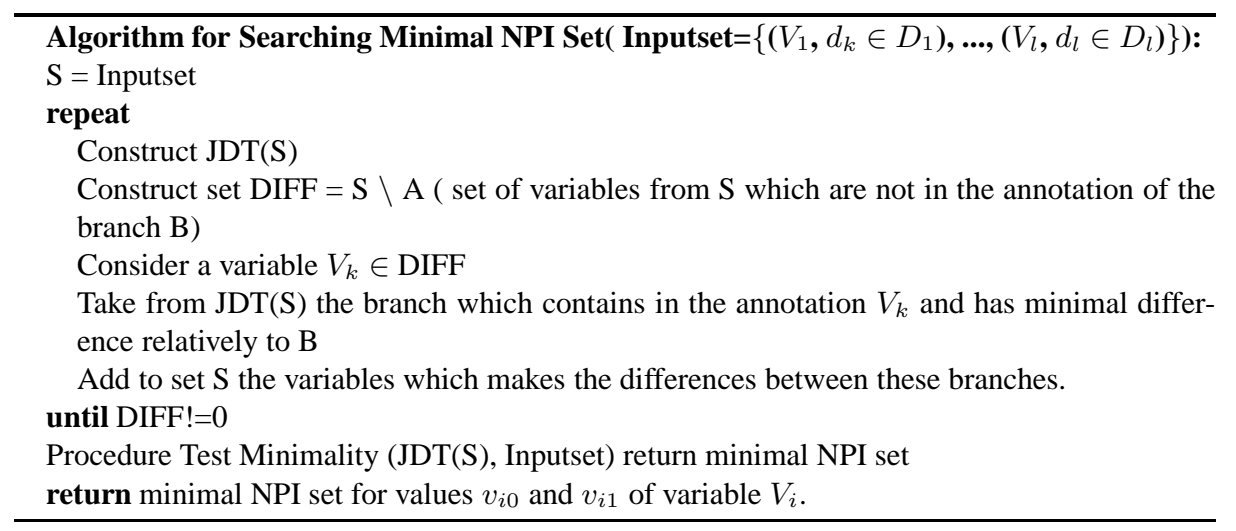

The procedure Test Minimality checks if the NPI set found is minimal and if not returns the minimal one. It is implemented as follows: once we have created the NPI we look for a minimal set by successively adding to the tree variables taken out from the set S. When nothing can be added to the tree without destroying the NPI, we have a minimal NPI set.

For a given input set there might exist more then one minimal NPI set. This algorithm is complete in the sense that if there exists minimal NPI set for a given input set, it will find at least one of them. ${ }^{3}$ Given a set $\mathrm{S}$ of size $\mathrm{s}$, the time complexity of the algorithm is $\mathrm{O}\left(\mathrm{n} \mathrm{s}(\mathrm{n}-\mathrm{s}) d^{2}\right)$, where $\mathrm{n}$ is the size of the CSP, and $\mathrm{d}$ is the size of the largest domain.

By applying the algorithm on the simple example from Figure 2 we obtain the following results:

\begin{tabular}{|c|c|}
\hline 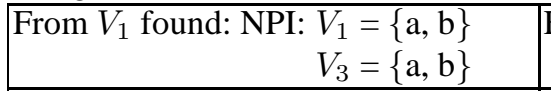 & From $V_{2}$ found: - \\
\hline $\begin{aligned} \text { From } V_{3} \text { found: NPI: } V_{1} & =\{\mathrm{a}, \mathrm{b}\} \\
V_{3} & =\{\mathrm{a}, \mathrm{b}\} \\
\text { NPI: } V_{3} & =\{\mathrm{c}\} \\
V_{4} & =\{\mathrm{c}, \mathrm{e}, \mathrm{f}\} \\
\text { NPI: } V_{3} & =\{\mathrm{a}, \mathrm{c}\} \\
V_{1} & =\{\mathrm{a}, \mathrm{b}\} \\
V_{4} & =\{\mathrm{c}, \mathrm{d}, \mathrm{e}, \mathrm{f}\}\end{aligned}$ & From $\begin{aligned} V_{4} \text { found: NI: } & V_{4}=\{\mathrm{e}, \mathrm{f}\} \\
\text { NPI: } & V_{3}=\{\mathrm{c}\} \\
V_{4} & =\{\mathrm{c}, \mathrm{e}, \mathrm{f}\} \\
\text { NPI: } & V_{4}=\{\mathrm{c}, \mathrm{d}, \mathrm{e}, \mathrm{f}\} \\
V_{1} & =\{\mathrm{a}, \mathrm{b}\} \\
& V_{3}=\{\mathrm{a}, \mathrm{b}, \mathrm{c}\}\end{aligned}$ \\
\hline
\end{tabular}

\footnotetext{
${ }^{2}$ The generalization to a set of variables as a starting point is straightforward and obtained by replacing the input with the set of variables and their corresponding values pair and followed by constructing the JDT for this input set.

${ }^{3}$ All can be found by eliminating sets as they are found.
} 


\section{Minimal NPI set characteristics:}

- In a CSP there are more minimal NPI sets for two given $v_{i 1}$ and $v_{i 2}$ values of a given variable $V_{i}$.

- To have a minimal NPI set by building the JDT for a given set of variables S we have to reach with all the variables in an annotation of the tree (end of one branch) such that all contain at least one value and at least one variable has more than one value (see Definition 5).

- The branch contains all the neighbor variables with at least one value; otherwise this NPI set does not interest as it means that there is no solution for the CSP.

- The set of the NPI variables has to be connected.

Proof:

Suppose we have a minimal NPI set $\mathrm{S}=\left\{V_{i}, V_{j}, V_{k}\right\}$ with NPI values NPI(S) $=$ $\left\{\left(V_{i},\left\{v_{i 1}, v_{i 2}\right\}\right),\left(V_{j},\left\{v_{j 1}, v_{j 2} \ldots\right\}\right),\left(V_{k},\left\{v_{k 1}, v_{k 2} \ldots\right\}\right)\right\}$ and variable $V_{k}$ has no constraints with any of the other two variables. As there are no constraints that means that all the values of $V_{i}$ and $V_{j}$ are compatible with all the values of $V_{k}$. That means that there exists NPI values for the set $\mathrm{S}^{\prime}=\left\{V_{i}, V_{j}\right\} \in \mathrm{S}$. That proves that $\mathrm{S}$ is not a minimal NPI set for the values $v_{i 1}, v_{i 2}$ of the variable $V_{i}$.

\section{Evaluation of NPI sets}

The performance of the algorithm for finding a minimal NPI set depends very much on the structure of the CSP problem. The following results are from tests analysing the number and coverage (size) of NPI sets detected w.r.t. varying CSP configuration (structure) by:

1. size of the problem: the number of variables.

2. the domains sizes of the variables.

3. the density of the problem: the ratio of the number of constraints relatively to the minimum and maximum number of constraints allowed in the given CSP, measured on a scale of $0.0-1.0$.

As the current application is over mutual exclusion CSPs, the tightness of the constraints, the fraction of the combinatorially possible pairs that are allowed by the constraint between two variables, is not taken into consideration. It will be an interesting issue in further work for more general CSPs.

The number and coverage of NPI sets found for solutions is a critical measure of the potential performance of the adaptation engine since it indicates the degree of freedom the engine will have in adapting a particular solution. It therefore gives a general indication of the general difficulty of the case adaptation problem for certain problem characteristics.

An accurate study of evaluation of NI sets has been done by Choueiry, Faltings and Weigel in [13]. This study measured the occurrence of NI sets depending on the configuration of the CSP and found that: 
- Only problems with low density allow NI values; the number of NI values become near to 0 for a density higher then 0.4 (this also corresponds to Benson and Freuder's results in [14]).

- For problems with low density the number of NI values increases with the number of resources.

- In general, the number of NI values decreases with the size of the problem.

These results indicate that NI interchangability is quite restricted in its domain of applicability and is one of the major reasons for attempting solutions based upon NPI sets (which are more likely to occur).

Performance is evaluated on randomly generated problems. Many CSP researchers use random instances to evaluate their constraint satisfaction algorithms.

Following the model of measuring the NI set as [13], we report the results only for problem sizes $n=10$ and $n=20$, while varying the density (dens-csp) in $\{0.1,0.2$, $\ldots, 1\}$ of the CSP and the maximum domain size dom-size $=\left\{\frac{n}{10}, \frac{2 n}{10}, \ldots, \frac{9 n}{10}, \mathrm{n}\right\}$. For each case, ten random problems were generated and then graphically represented by considering the measures described below.

Lets consider the CSP problem $\mathrm{G}=(\mathrm{V}, \mathrm{E})$ as a constraint graph where $\mathrm{V}$ represents the vertices (variables) and E edges (constraints), and

- The density of the problem is: dens-csp $=\frac{e-e_{-} \min }{e_{-} \max -e_{-} \min }$, where e represents the number of edges in the current CSP, e_min $=n-1$ and $\mathrm{e} \_\max =\frac{n(n-1)}{2}$, where $\mathrm{n}$ is the size of the problem CSP.

- $\operatorname{nNPI}\left(V_{i}\right)$ is the number of minimal NPI sets for variable $V_{i}$ where we computed a minimal NPI set for each pair of values from the domain of $V_{i}$.

- $\operatorname{avNPI}\left(V_{i}\right)=\frac{\sum_{k=1}^{n N P I} \text { size }(k)}{n N P I}$ is the average size of an minimal NPI set for variable $V_{i}$, where size(k) representes the number of variables in the current NPI set.

- $\|V\|$ is the number of variables which has minimal NPI sets over their entire domain (all possible pair values).

In the following we introduce the three criteria we used to measure the existence and maximality of NPI sets in the CSP.

Coverage: maximality of the NPI sets. $\mathrm{m} 1$ measures the "maximality" of the neighbourhood partial interchangeability (NPI sets) in the sense that we computed the average size of the minimal NPI set in a given CSP:

$$
m 1=\frac{\sum_{k=1}^{\|V\|} a v N P I(V k)}{\|V\|}
$$

We have the graphical representation in the figure 4 for problems of size 10 and in the figure 5 for problem of size 20 . We can see that for problems with low density the coverage tends to 0 . This means as expected that in low density problems exchanging the values of one variable do not propagate too much in the CSP (corresponds to NI, interestingly the threshold corresponds to the results cited above). This indicates that NI adaptation could be successfully applied here. Coverage increases with the number of resources. 
The two metrics applied to measuring the number of NPI occurrences were as follows:

Occurrence 1: existence of the NPI sets. 2 measures the "occurrence" of NPI sets in a given CSP in the sense that it computes how many variables have minimal NPI set in rapport to the size of the problem.

$$
m 2=\frac{\|V\|}{n}
$$

Looking at the figures 6 and 7 we have the proof that NPI occurs often in any CSP configuration and increases with the density of the CSP as well as with the number of resources.

Occurrence 2: existence of the NPI sets. m3 measures the "occurrence" of NPI sets in the sense that it computes the average number of NPI sets per variable.

$$
m 3=\frac{\sum_{k=1}^{\|V\|} n N P I(V k)}{n}
$$

The average number of NPI sets per variable depends in the same way as Occurrence 1 on the configuration of the CSP, but is a better indicator w.r.t the density of the problem and highly dependent on the number of resources, see 8 and 9.
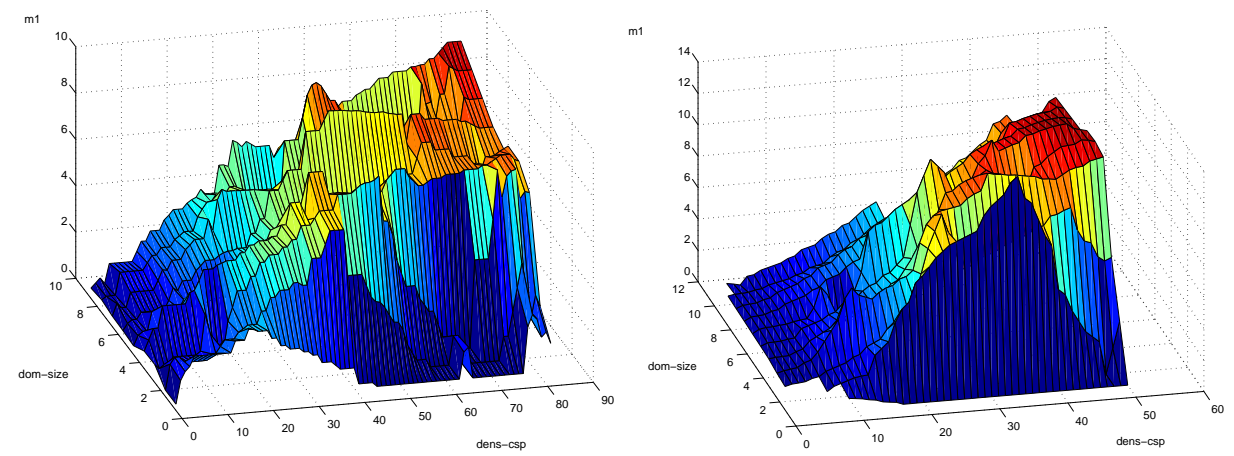

Figure 4: Coverage of NPI sets for random Figure 5: Coverage of NPI sets for random generated CSPs with size 10. generated CSP with size 20.
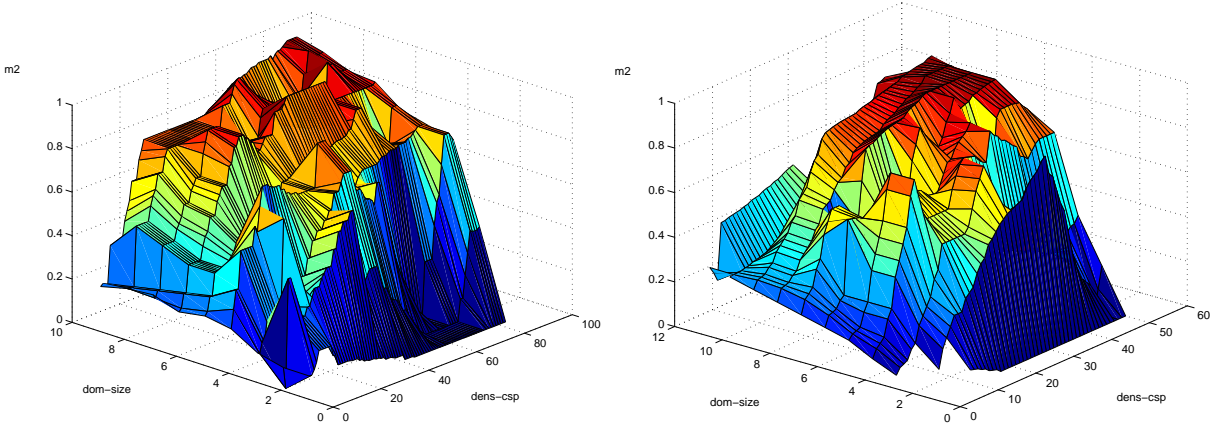

Figure 6: Occurrence of NPI sets per vari- Figure 7: Occurrence of NPI sets per variable in random CSPs with size 10. able in random CSPs with size 20. 


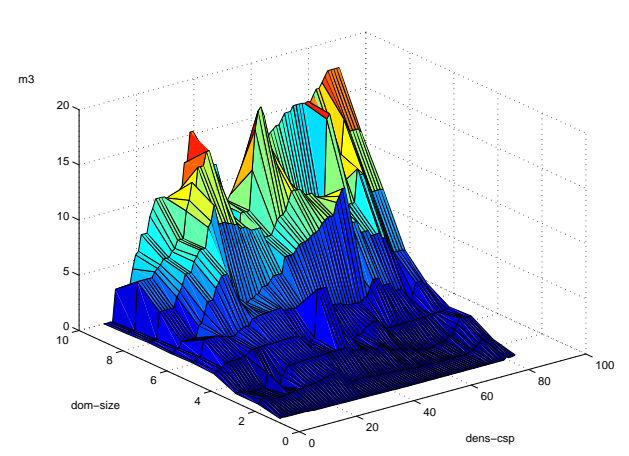

Figure 8: Average number of NPI sets per Figure 9: Average number of NPI sets per variable in random CSPs with size 10 .

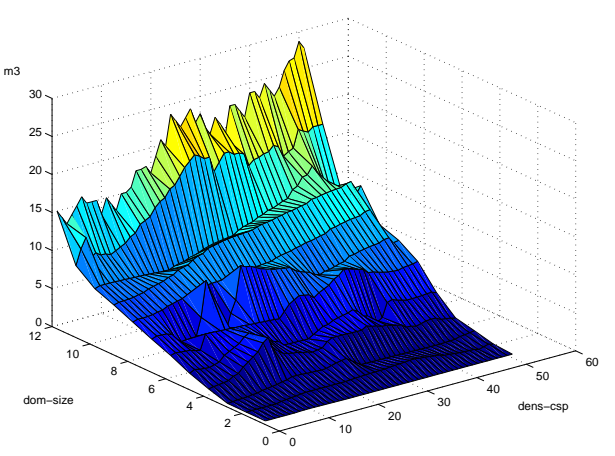

variable in random CSPs with size 20.

From the results we can conclude that on higher density problems the occurrence of NPI increases and thus the range of the adaptation increases as well with it, but in the same time the coverage is enlarged over the variables of the CSP. When the coverage enlarges, the effect of a change propagates further. Thus at a certain point, searching the NPI sets should be stopped. When the NPI set becomes too large, it might not bring any profit anymore as it would be equivalent to or even more costly than solving the problem from scratch. In a further work we will study how much a change should maximally propagate in a CSP to keep NPI profitable.

\section{Related Work}

There are some other approaches to seeing the adaptation problem as a constraint satisfaction problem. The solution of a new problem is built by satisfying the new constraints and by transforming a memorised solution. Hua, Faltings and Smith [15] proposed a case-based reasoner for architectural design where constraints restrict numerical relationships among dimensions. CADRE introduced the concept of dimensional reduction: before attempting to adapt a case, it constructs an explicit representation of the degrees of freedom available for adaptation. However, CADRE defined this approach only for numeric constraints. The adaptation method based on dimensional reduction has been tested successfully in the IDIOM project [16].

In the domain of discrete variables, the adaptation space is less obvious. We proposed here a method based on interchangeability which localise changes in discrete spaces and thus offers a reliable method for determining the closest solution.

Another approach for adaptation based on constraints over discrete domains was done by Purvis and Pu [17] in a case-based design problem solving domain. Their methodology formalises the case adaptation process in the sense of combining multiple cases in order to achieve the solution of the new problem, by applying repair-based CSP algorithm [18]. Our method do not rely only on the knowledge accumulated in the case base but also on the domain knowledge formulated as CSP. The approaches are different as by their method the constraints between specific values of the variables are stored in the case and we consider in the case only the solution of the new problem, while the 
constraints between variables are hold in an external module which contains the domain knowledge. We believe that our approach gives more flexibility to adaptation module but we will study in the future how to combine the two methods for improving the adaptation process.

\section{Conclusions}

Up until now, interchangeability has mostly been applied as a technique for enhancing search and backtracking, see [19] and [20]. It had not been effectively exploited as a technique for updating solutions. In this work we have studied how interchangeability can be applied for updating solutions with the goal of using it further in case adaptation. The work makes the following main contributions:

- A complete algorithm for detecting NPI sets which can be used to provide more flexible adaptation than NI based approach would.

- An evaluation of how far changes propagate in solutions to be adapted according to problem structure (investigating impact of density and size of the problem.) indicating:

- Strong dependency on the density parameter of the CSP where NPI are more frequent in CSPs with high density.

- Weak dependency on the domain sizes where NPI increases with the number of resources.

- Presentation of NPI based adaptation as a good candidate for general case adaptation strategies on a large class of problems

On the basis of this work we believe that Neighbourhood Partial Interchangeability has strong potential as an important strategy for identifying classes of equivalent solution as a basis for adaptation.

\section{Further work}

Interesting future directions include:

- Detecting minimum set of changes in a given solution (the smallest from all the possible minimal NPIs).

- It is claimed that random problems are not very relevant in proving the results; so we consider as a step forward testing these algorithms and strategies on real-world problems.

- Improving the optimisation strategies for the finding minimal NPI set algorithm.

- Developing new algorithms for finding NPI values by decomposing the problem into different subproblems.

- Investigating the distribution of NPI sets in a solution by size of coverage (small coverage sets are useful to minimise overhead in adaptation).

- Classifying case bases of solutions by how re-usable ("adaptable") their solutions are according to density and other CSP configuration measures. 


\section{Acknowledgements}

The authors are indebted to Steve Willmott and Djamila Sam-Haraud for valuable discussions and comments.

\section{References}

1. D. Leake. Case-Based Reasoning: Experiences, Lessons and Future Directions. In AAAI Press, 1996.

2. E. Freuder. Constraint-based Reasoning. MIT Press, 1994.

3. Van Hentenryck. Generality versus Specificity: An Experience with AI and OR Techniques. In AAAI-88: Proceedings National Conference on Artificial Intelligence, pages 660-664, 1988.

4. R. Dechter Y. El Fattah. Diagnosing tree-decomposable circuits. In Proc. of the 14 th IJCAI, pg. 1742-1748, 1995.

5. H. Krautz and B. Selman. Planning as Satisfiability. In Proc. of the 10 th Ecai, pages 359-363, Vienna, Austria, 1992.

6. M. Wallace. Applying constraints for scheduling. In Constraint Programming, volume 131 of NATO ASI Series Advanced Science Institute Series. Springer Verlag, 1994.

7. A. Mackworth. Constraint-based Design of Embeded Intelligent Systems. In Constraints 2(1), pages 83-86, Vienna, Austria, 1992.

8. R. Weigel and B. Faltings. Interchangeability for Case Adaptation in Configuration Problems. In Proceedings of the AAAI98 Spring Symposium on Multimodal Reasoning, Stanford, CA, TR SS-98-04., 1998.

9. E. C. Freuder. Eliminating Interchangeable Values in Constraint Satisfaction Problems. In In Proc. of AAAI-91, pages 227-233, Anaheim, CA, 1991.

10. B. Y. Choueiry and G. Noubir. On the Computation of Local Interchangeability in Discrete Constraint Satisfaction Problems. In Proc. of AAAI-98, pages 326-333, Madison, Wiscowsin, 1998.

11. Z. Ruttkay. Fuzzy Constraint Satisfaction. In 3rd IEEE Int. Conf. on Fuzzy Systems, pages 1263-1268, 1994.

12. N. Neagu and B. Faltings. Constraint Satisfaction for Case Adaptation. Proceedings of the Workshops at ICCBR'99, pages III-35-III-41, 1999.

13. B. Choueiry B. Faltings, R. Weigel. Abstraction by Interchangeability in Resource Allocation. In Proc. of the 14 th IJCAI-95, pages 1694-1701, Montreal, Canada, 1995.

14. B.W. Benson and E. Freuder. Interchangeability Preprocessing can Improve Forward Checking Search. In Proc. of the 10 th Ecai, pages 28-30, Vienna, Austria, 1992.

15. B. Faltings K. Hua and I. Smith. CADRE: case-based geometric design. In Artificial Intelligence in Engineering 10, pages 171-183, 1996.

16. C. Lottaz I. Smith and B.Faltings. Spatial composition using cases: IDIOM. In Proc. of the 1st International Conference in CBR, pages 88-97, 1995.

17. L. Purvis and P. Pu. Adaptation using Constraint Satisfaction Techniques. In Proc. of the 1st International Conference in CBR, pages 88-97, 1995.

18. A. Philips S. Minton, M. Johnson and P. Laird. Minimizing Conflicts: A Heuristic Repair Method for Constraint Satisfaction and Scheduling Problems. In Artificial Intelligence 58, pages 88-97, 1995.

19. E. C. Freuder and D. Sabin. Interchangeability Supports Abstraction and Reformulation for Multi-Dimensional Constraint Satisfaction. In In Proc. of AAAI-96, pages 191-196, Portland, 1991.

20. A. Haselbock. Exploiting Interchangeabilities in Constraint Satisfaction Problems. In Proc. of the 13 th IJCAI, pages 282-287, 1993. 